\section{Cone beam CT in dental practice}

\author{
A. Dawood, ${ }^{1}$ S. Patel ${ }^{2}$ and J. Brown ${ }^{3}$
}

CORE VERIFIABLE CPD PAPER
IN BRIEF
- $\mathrm{CBCT}$ is a relatively new technology to dentistry, used for the 3D imaging of the teeth and jaws.
- Radiation dose to the patient is much less than for conventional CT scanners, but still higher than for conventional 2D dental imaging.
- Training is crucial for all members of the dental team involved in CBCT radiography and radiology.

Cone Beam Computed Tomography is a relatively new three-dimensional imaging technology, which has been specifically developed for imaging of the teeth and jaws. The aim of this paper is to acquaint the dental team with various forms of this technology and its potential applications. An understanding of the underlying principles will allow the users of this technology to tailor the imaging protocol to the patient's individual needs to achieve appropriate imaging at the lowest radiation dose.

\section{INTRODUCTION}

Cone Beam Computed Tomography (CBCT) is a relatively new technology to dentistry, used for the three-dimensional imaging of the teeth and jaws (Fig. 1). CBCT is a result of dramatic advances in computer and electronic technology and (along with similar advances in scanning and manufacturing) is one of the key components in the rapidly evolving field of digital dentistry. It is becoming widely available and has applications in implant dentistry, endodontics and oral surgery.

This paper is intended to introduce CBCT technology and highlight the differences between CBCT and conventional Computed Tomography (CT). It will examine how CBCT can be used to best effect, or unintentionally abused when imaging the dental patient.

\section{BACKGROUND}

Intra-oral and extra-oral radiographs captured on plain films and digital sensors are two-dimensional shadowgraphs, which

\footnotetext{
"Dawood \& Tanner Dental Practice, 45 Wimpole Street, London, W1G 8SB; ${ }^{2}$ Endodontic Postgraduate Unit, King's College London Dental Institute, Floor 25, Tower Wing, London SE1 9RT; ${ }^{3}$ Department of Oral and Maxillofacial Radiology, King's College London Dental Institute, Floor 23, Tower Wing, Guy's Hospital, London SE1 9RT

*Correspondence to: Mr Andrew Dawood

Email: andrewdawood@hotmail.com
}

\section{Refereed Paper}

Accepted 6 May 2009

DOI: $10.1038 /$ sj.bdj.2009.560

${ }^{\oplus}$ British Dental Journal 2009; 207: 23-28 represent the absorption of the incident $\mathrm{X}$-ray beam as it penetrates the different structures before exposing the image receptor. ${ }^{1}$ The amount of useful information gained from these radiographic techniques is limited as the complex anatomical structures between the X-ray source and image receptor are compressed into this two-dimensional shadowgraph., ${ }^{2,3}$ In addition there is often a degree of geometric distortion and magnification of the resulting image ${ }^{4}$ (Fig. 2a). Furthermore, overlying anatomical noise may result in difficulty interpreting radiographs.,

The interpretation of the radiograph is dependent on the clinician's appreciation of the limitations of conventional radiography as well as their knowledge and experience in assessing these two-dimensional shadowgraphs. ${ }^{3}$

In conventional tomography a sectional image may be obtained by moving the X-ray source and film in opposing directions during an exposure. Structures appear blurred if not in the focal plane and relatively sharper when in the focal plane, particularly if the relative movement is complex, for example spiral tomography. Dental Panoramic Tomography relies on a relatively complex interrelationship between the movement of source, film and a rectangular collimator to achieve a tomographic projection of the curved arch of the jaws. ${ }^{7}$

In these forms of tomography the complex motion of X-ray source and receptor means that the equipment is mechanically

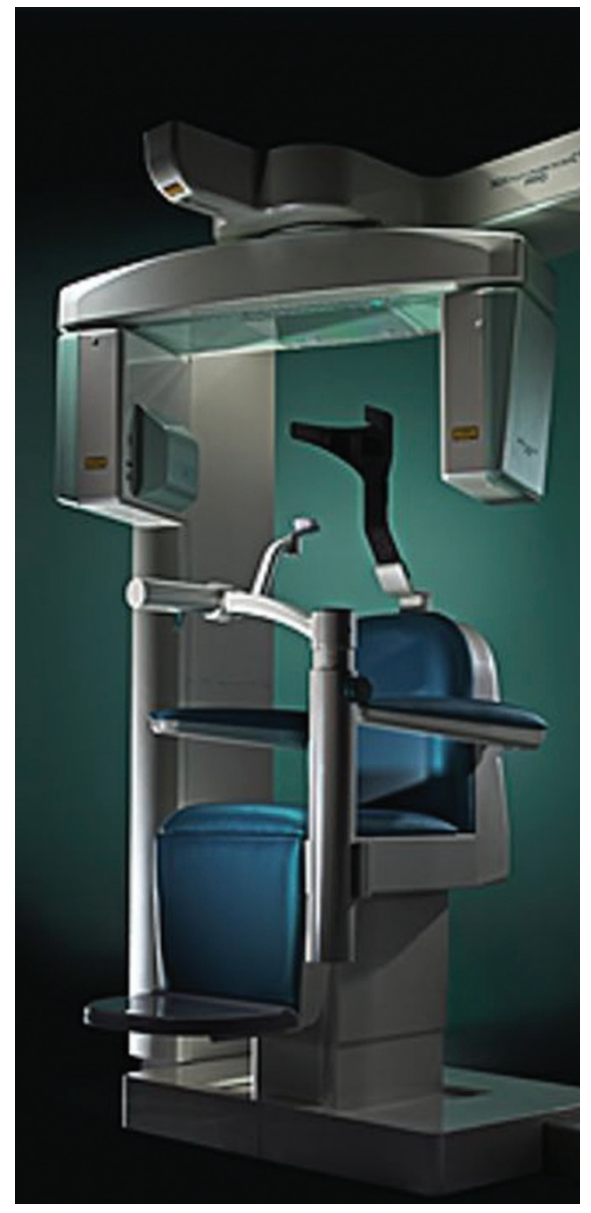

Fig. 1 An Accuitomo F170 (Morita corp, Kyoto Japan), a CBCT scanner with a variable FOV

sophisticated, though the image receptor itself is relatively unsophisticated.

CT provides three-dimensional imaging and has been used to overcome the inherent problems with conventional twodimensional radiographic techniques (Figs 


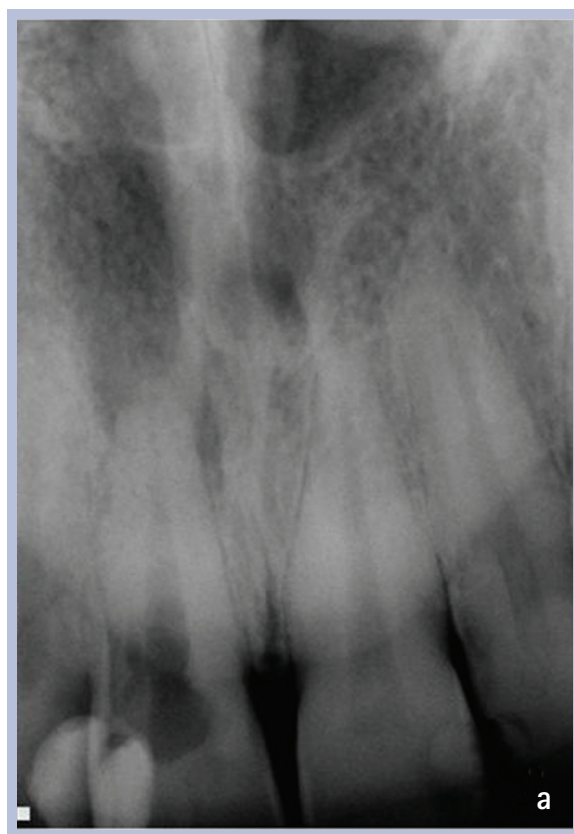

Fig. 2 The benefits of three-dimensional imaging. (a) An intraoral radiograph of an asymptomatic central incisor fails to show the true nature of the extensive lesion compared with re-sliced CBCT images; (b) coronal (c) sagittal and (d) axial

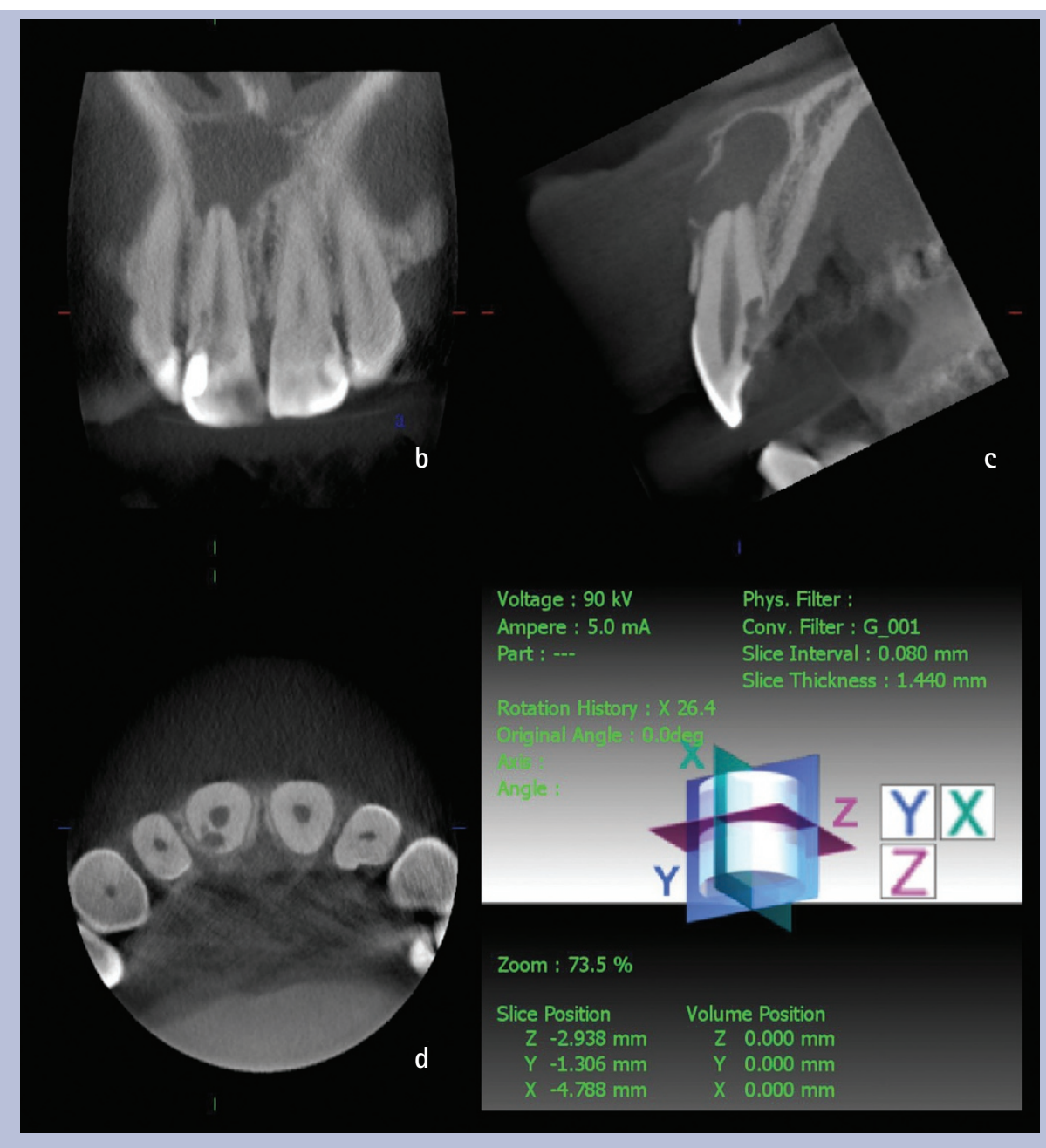

reconstruct using a 'mini' computer. It is interesting to note that as imaging technology has evolved the emphasis has shifted from complex to simple mechanisms and from simple film-based image acquisition to sophisticated electronic data acquisition and computer processing.

CT has continued to evolve; contemporary multi-slice technology allows the acquisition of large volumes of data, capturing a large section with each revolution of the gantry in less than a second. Although offering spectacularly high speed imaging of both hard and soft tissues, X-ray dose is high, the equipment exceptionally expensive and generally only found in hospital settings.

\section{Cone Beam Computed Tomography}

CBCT is a low dose scanning system, which has been specifically designed to produce three-dimensional images of the maxillofacial skeleton. ${ }^{9,10}$ CBCT scanners use back-projection reconstructed tomography to acquire data of the area of interest through a single or partial rotation of the conical X-ray beam and reciprocal image receptor. Remarkably, in most systems scan times of less than 20 seconds may be achieved using commonly available personal computers to process the data. Thus a principle difference between CT and CBCT is the method by which data are gathered - while CT acquires image data using rows of detectors, CBCT exposes the whole section of the patient over one detector (Fig. 3); these data are then used to generate individual slice images.

As the patient is stationary and the motion of the gantry is a simple rotation, the main complexity of the CBCT system lies in the detector and the data processing technology.

The acquired images are called twodimensional projections. Reconstruction algorithms are used to convert them into a three-dimensional data set.

The CBCT volumetric data set is usually reconstructed in orthogonal orientations to allow viewing of the images in the axial, sagittal and coronal planes. Visualisation software allows the brightness and contrast 


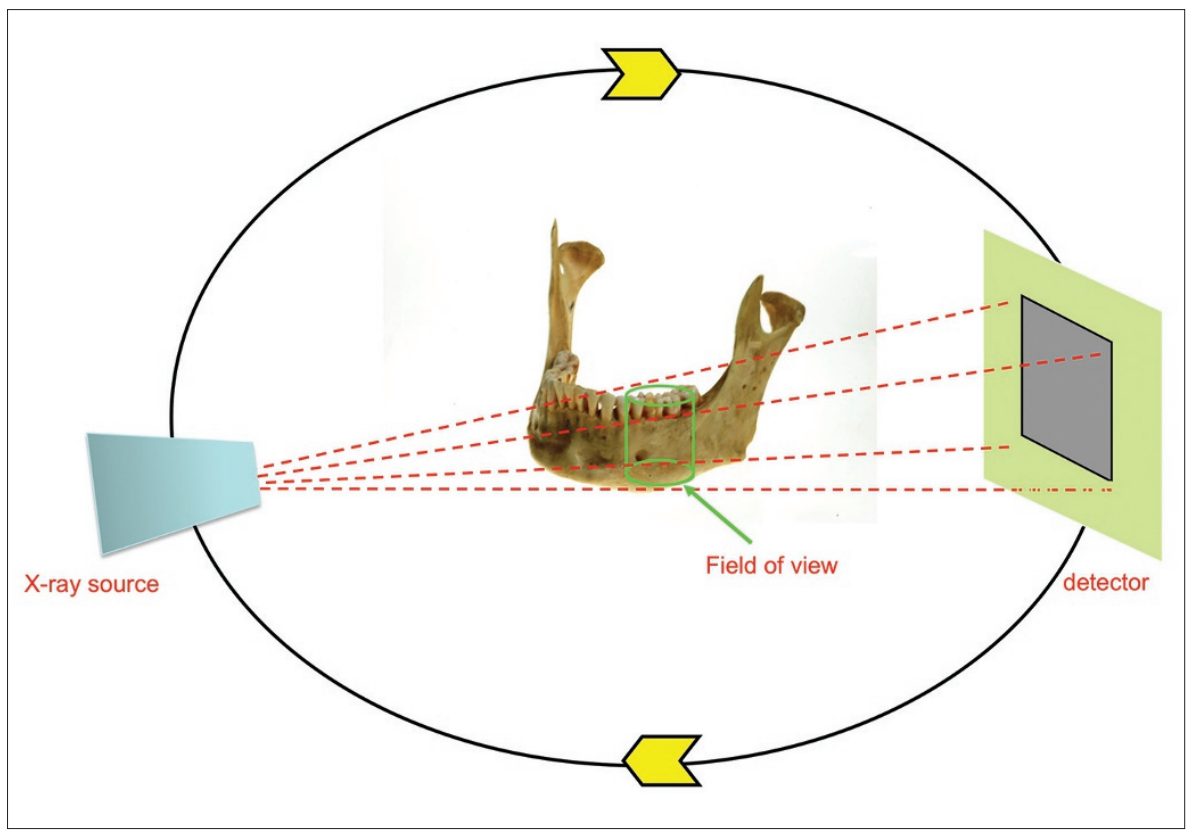

Fig. 3 Source and opposing flat panel detector rotating around the patient's jaw, capturing image data in one sweep

of the greyscale images to be adjusted. When viewing both CT and СBCT data it is common to speak in terms of window and level; the 'window' describes the range of greyscale values that will be visualised, while the 'level' specifies the mid-value at which the window will lie. Post-processing software may also be used to 'render' the volume thus allowing the three-dimensional visualisation of the structures, such as the bone surface and teeth (Fig. 4).

CBCT scanners use comparably less sophisticated hardware and software than CT scanners, resulting in simpler, more compact and less expensive machines, but produce comparable, or better images of the hard tissue structures of the jaws and skull. ${ }^{11,12}$ These advantages make CBCT technology particularly well suited to hard tissue imaging and dentistry. As the X-ray dose is so much lower than for conventional CT scanners, it is difficult to justify the use of conventional CT for elective dental and maxillofacial procedures when CBCT is available.

However, as outlined below, a disadvantage of CBCT is that numerous factors may combine to affect the linearity of the exposure on the flat panel sensor, ${ }^{13}$ making it difficult to directly apply the Hounsfield scale across the full expanse of the scanned volume (Figs 5a-b). It should also be stressed that soft tissue detail is not displayed as with conventional CT. Thus CBCT would not be particularly suitable for examining lesions involving both soft tissues and bone, unless only the bony element is being investigated.

\section{DENTAL CBCT}

CBCT technology is becoming more widely available and less costly; CBCT scanners are available from most dental X-ray equipment manufacturers in a wide variety of formats with various different attributes.

For the most part the patient is examined standing or seated, and the machines have the footprint (though not necessarily the capability) of a panoramic unit.

The captured volume of data is called the 'field of view' (FOV). Scanners are available to image volumes ranging from the whole skull to just a small volume incorporating a few teeth. Not surprisingly, as larger volumes are exposed, or resolution is increased, X-ray dose will increase.

Similarly the size of the digital files, which is large anyway, will increase when using higher image resolution and larger FOV. Large digital scans are cumbersome to process and view and eventually require more storage space. Security of data storage should also be considered, as a matter of clinical governance.

\section{Field of view}

Ideally the FOV should be adjustable in height and width to limit radiation exposure to the area of interest only, therefore reducing the radiation exposure to the patient. ${ }^{14,15}$

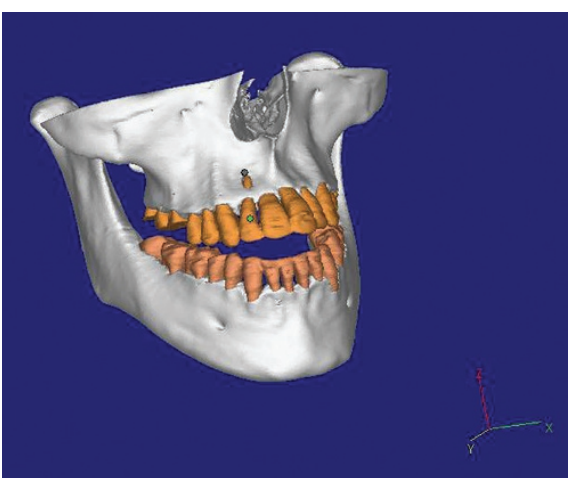

Fig. 4 Rendering allows visualisation of the three-dimensional structures within the field of view, such as the bone surfaces and teeth
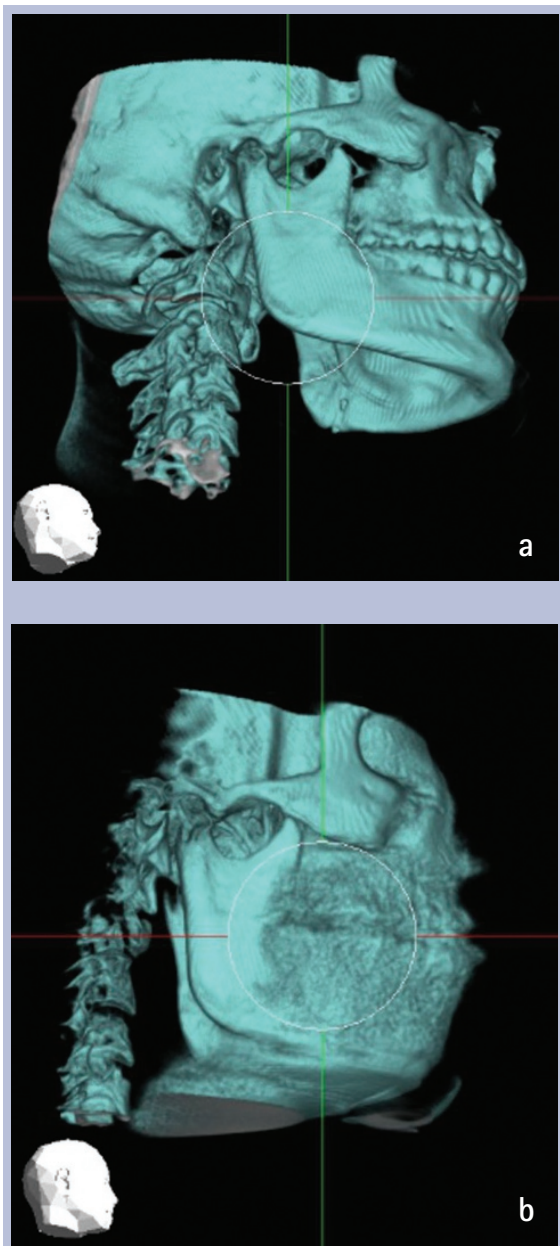

Fig. 5a-b These rendered 3D images show how different calibration parameters will affect the way that the same structure is visualised in 3D

The capacity to control the FOV is exceptionally important in terms of limiting X-ray dose. When selecting a CBCT scanner it is important to choose equipment that has a FOV appropriate to the intended usage. Some early scanners did not have the option to alter the FOV, making their use inappropriate in certain situations. For example, it is inappropriate to 
scan the entire maxillofacial region to aid diagnosis and plan treatment for single unit implant placement or endodontic treatment. Furthermore, exposure of a wide FOV places additional responsibilities on the dental practitioner - these scans may cover areas of the spine, maxillofacial skeleton and skull base, which the practitioner will have a duty to evaluate and report on (Fig. 6), but may lack the necessary experience to do so.

Using a smaller FOV also reduces the amount of data produced, as fewer voxels are recorded. This has a positive impact on the need for data storage capacity and the speed of data processing and onscreen data manipulation. It is therefore recommended to use high-resolution on small FOV only.

\section{Resolution}

The resolving power of an imaging medium is its ability to display detail and is commonly defined by the ability to distinguish line pairs per $\mathrm{mm}$ in a specially designed test tool of alternating lead and plastic slats.

In CBCT the resolution of the image is dependent on several factors. These include the quality and resolution of the flat-panel detector (or photomultiplier tube, as found on earlier machines), and the number and rotational spacing of the individual basis images from which the three-dimensional volume of data is generated. Other factors affecting the resolution include the sophistication of the reconstruction algorithm in the software, the power of the $\mathrm{X}$-ray source, the refinement of the projection geometry and resolution of the viewing monitor. Increased resolution usually comes at the expense of an increased radiation dose to the patient, as a result of longer exposure times to acquire more 2D projections to contribute to a more detailed reconstruction.

While a higher dose, higher resolution scan may improve the aesthetics of the resulting data, it may be possible to fully achieve the objectives of the examination using a lower resolution setting or by reducing exposure parameters ${ }^{14}$ to achieve a lower dose to the patient. Thus to limit the patient's radiation exposure it is essential to tailor the resolution to meet the demands of each unique case being managed (Table 1).
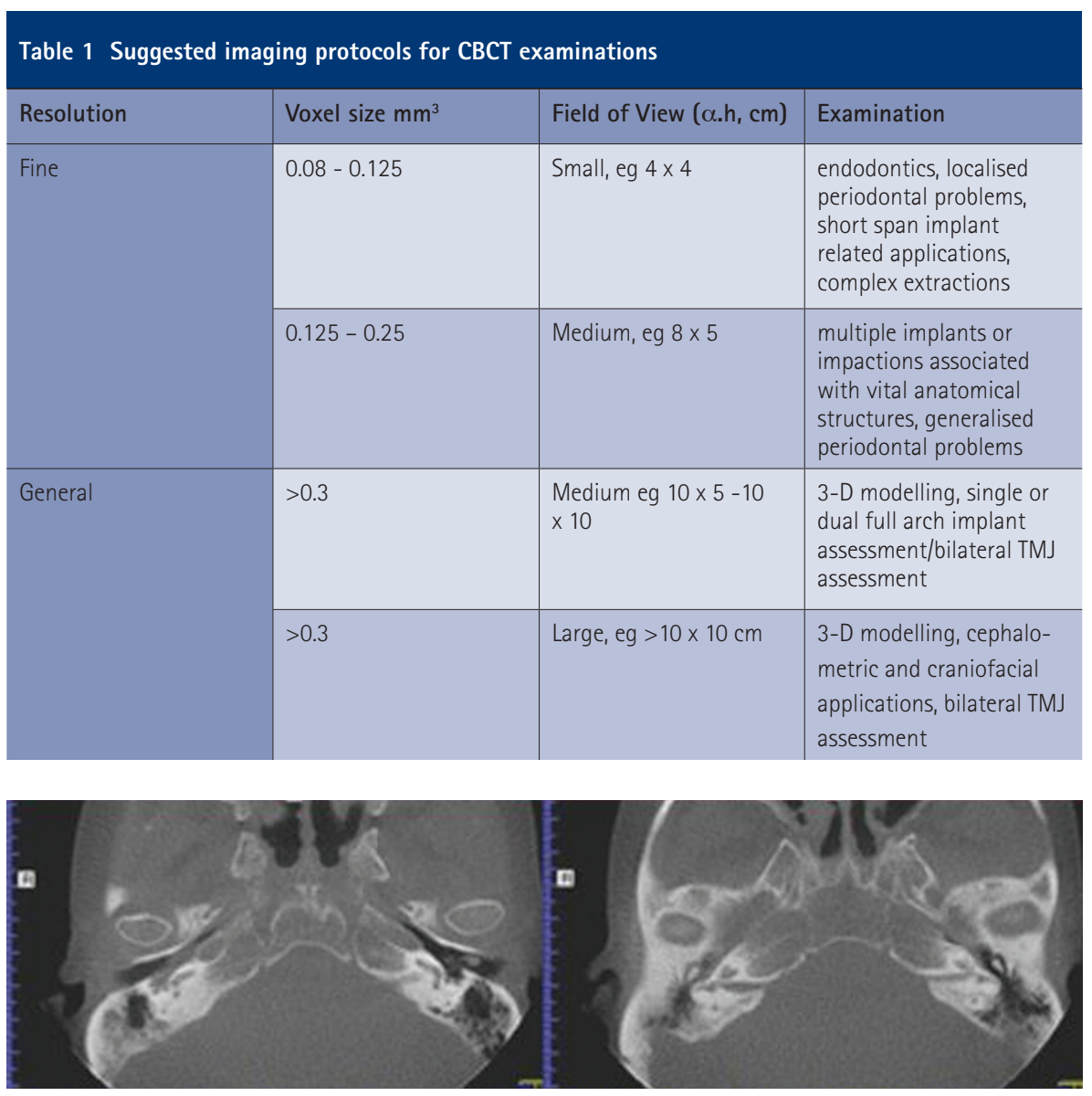

Fig. 6 Can a dentist identify the right middle ear and mastoid infections diagnosed as a coincidental finding in a large FOV examination of the maxilla?

\section{Dose considerations}

Occupational exposure from CBCT should not be an issue when such equipment is correctly installed. The CBCT supplier and the Radiation Protection Advisor (RPA) should collaborate to design the facility in which the CBCT scanner is to be housed with appropriate shielding to protect staff during exposure, as is already required by the Ionising Radiations Regulation (1999). ${ }^{16,17}$ CBCT units are capable of greater power and X-ray scatter than conventional dental $\mathrm{X}$-ray units and would normally require a dedicated room where the operator may stand outside or behind a suitable screen of brick or lead. During a conventional dental intra-oral X-ray exposure, many practitioners do not use shielding but stand a safe distance of over $1.5 \mathrm{~m}$ behind or to the side of the machine. Applying this principle, an operator would need to stand at least $8 \mathrm{~m}$ from a CBCT machine.

The average exposure to a patient during a CT examination is commonly estimated and presented as the computed tomography dose index (CTDI). All modern CT equipment must display this figure, allowing for the estimation of the effect of different scan protocols on dose. However, this method of dose estimation is not available with all CBCT apparatus. An alternative approach for CBCT is to use the "dose area product' (DAP), which estimates the exposure to the patient by directly measuring the incident X-ray beam.

It is important to note that while the output of an X-ray source is measured in Gray, the biological effect of the beam varies with the age and gender of the patient and the radio-sensitivity of the exposed tissue. This 'biological' effect is measured by the 'effective dose' in Sieverts. A recent 'effective dose' survey showed CBCT units delivered a broad range of doses (dependent on machine, field size, resolution, etc) of between $13 \mu \mathrm{Sv}$ (minimum dose, small volume) and $82 \mu \mathrm{Sv}$ (maximum dose, large volume) which compared favourably with radiation dose inflicted by multi-slice CT (MSCT) of between $474 \mu \mathrm{Sv}$ and $1,160 \mu \mathrm{Sv}$ for mandibular and full head scans respectively. To put these measurements into perspective, panoramic doses have recently been found to range between 3-24 $\mu \mathrm{Sv} .{ }^{18,19}$ 
As already discussed, patient radiation dose may be minimised by matching the FOV and resolution to the intended usage, keeping the FOV as small as possible and minimising exposure of radiosensitive tissues. Adolescents and children are much more sensitive to radiation exposure and so prescription of CBCT examinations for these individuals needs to be highly focused towards the need of the individual.

The salivary and thyroid glands are radiosensitive organs that may be unnecessarily exposed by direct radiation, or indirectly from radiation scatter from an unnecessarily large FOV, or excessively detailed (ie high resolution) scan. ${ }^{13}$

It is possible to generate a 'true' dental panoramic tomograph (DPT) by using a specifically designed 'dual-purpose' CBCT scanner, if it is fitted with the appropriate hardware. CBCT data can be reconstructed to produce DPT-like images. However, this examination gives the patient a higher radiation dose and cannot be justified as an alternative approach to obtain a conventional DPT.

\section{Data presentation and viewing}

The appearance of scan data is much affected by the overall exposure parameters, the efficiency of the detector and the reconstruction. Reconstructing thicker slices will give a lower contrast but a less noisy appearance, while thinner slices will appear to have more contrast and detail, but are noisier. Lower dose exposures will typically be noisier than higher dose exposures. Slower scan times may allow for higher resolutions but will be more susceptible to movement artefact. For threedimensional modelling, there is typically a need for a slice thickness $<0.5 \mathrm{~mm}$.

Although individual reconstructed CBCT images may be printed on film or paper, most viewing software allows for a plethora of changes to the reconstruction, each of which may have a significant effect on the viewed image. This means that viewing data on-screen is important and cannot be replaced by a print-out.

Computer processing is essential for СBCT so software for the reconstruction and display of images may be considered as an integral part of the apparatus. The software will have a pronounced effect on the appearance of the scan - therefore in many respects the software that works with the scanner is as important as the hardware.

Typically software allows reconstruction of the scan data in three orthogonal planes and frequently along a curved plane as a 'panoramic' reconstruction. Changes to the window and level will change the emphasis of what is visualised. When choosing a particular scanner it is important to ensure that its viewing software is appropriate to the needs of the practice; this includes the ability to view data on different workstations and in more than one location.

The software will typically also export data to third party software for implant, maxillofacial or orthodontic treatment planning. Along with the increase in the availability of CBCT scanners, development in software and rapid prototyping technology continues apace, particularly in the field of implant dentistry and surgical planning, leading to many new treatment protocols for surgery (Figs 7-8).
The electronic display used will also have a significant effect on the perceived image. Perception will be improved by using a high quality monitor viewed in muted lighting conditions.

\section{Reporting}

The current UK IRMER 2000 legislation (IRMER) ${ }^{16}$ places a duty on all practitioners to ensure that radiographic images are fully evaluated, with abnormal or pathological findings recorded into the patient's notes. The law lays this duty on the 'Operator' and 'Legal Person' (the owner of the X-ray installation), to ensure that a radiographic report is made. If an adequate clinical evaluation of the image is unlikely to be made, that the exposure would not be justified and the radiograph should not go ahead.

CBCT images of the immediate dento-alveolar area will provide dentists with images of a region that they are well qualified to
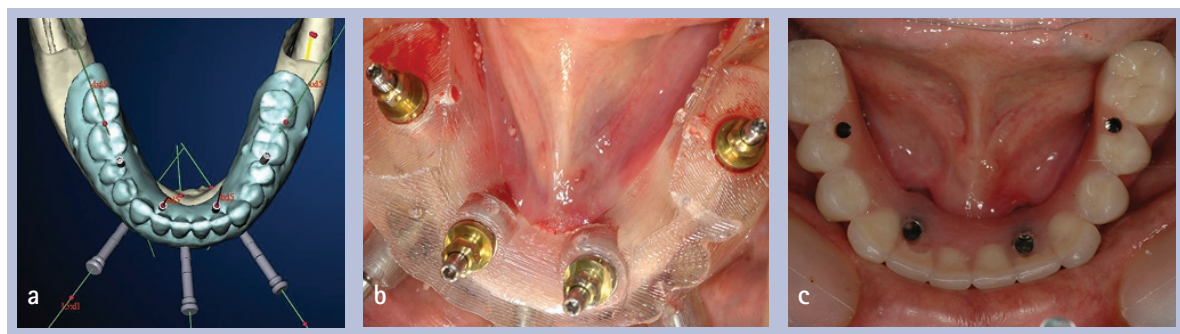

Fig. 7 Maxillofacial and implant planning software allows the surgeon to interact with scan data to visualise planned surgery on a rendered virtual 3D model of the jaw. The precise position of a dental implant in the ridge may be modelled. Combining the visualisation of a virtual model of the jaw, with computer aided design software it is possible to plan implant placement and make constraining drill-guides using 3D manufacturing techniques. When fitted to the jaw, the guides precisely constrain implant site preparation, allowing implants to be placed using a minimally invasive approach directly into pre-planned positions. The precise position of a dental implant in the ridge may be modelled. Combining the visualisation of a virtual model of the jaw, with computer aided design software it is possible to plan implant placement and make constraining drill-guides using 3D manufacturing techniques. When fitted to the jaw, the guides precisely constrain implant site preparation, allowing implants to be placed using a minimally invasive approach directly into pre-planned positions. Implant planning in a virtual environment (a), a drill guide in place (b), and a robust temporary resin bridge which was prefabricated on the basis of the computer plan, still in function three years post operatively (c)
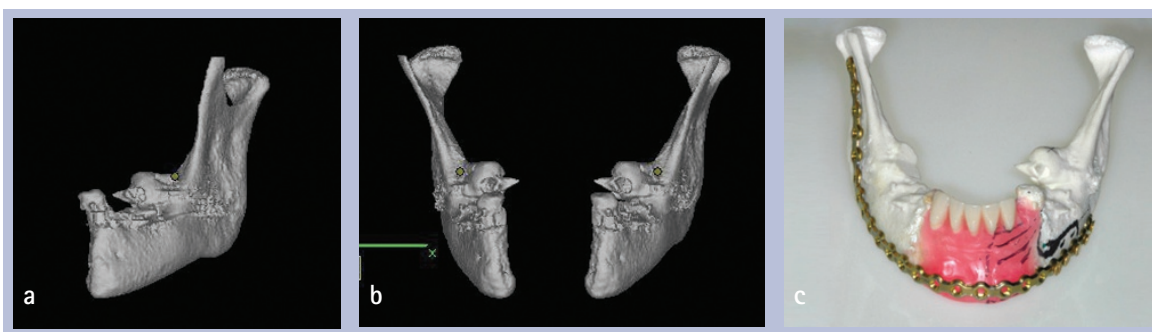

Fig. 8 Reconstructive surgery in a virtual environment: the left mandibular fragment has been mirrored and repositioned prior to reconstruction in order to simulate the contour of the mandible ( $a$ and $b$ ). Conventional modelling techniques have been used to approximate a shape for the missing anterior segment and pre-bend a suitable titanium fixation plate on a rapid prototype model prior to implant surgery (c) 
report on. Wider FOVs do, however, capture the skull base, sinuses and cervical spine, which are not normally within a dentist's area of expertise and this would require reporting by a dento-maxillofacial or head and neck radiologist. Restricting the extent of the FOV to the particular region of interest has the benefit of reducing the need for the practitioner to seek assistance to report regions that are beyond their competence. Figure 6 shows a right middle ear and mastoid process infection; an abnormality in an area not normally seen on dental films, but which now comes into the CBCT field of view and requires a report.

\section{Training for work with CBCT}

The law also requires anyone using $\mathrm{X}$-ray equipment to be 'adequately trained' for the role they play. In the case of radiographic exposures the law recognises different roles; the 'referrer', the 'IRMER practitioner' and the 'operator'. Each has a specific role and needs to be prepared by suitable training to fulfil that role. Rather uniquely, a dentist may find he or she fulfils all these roles.

The 'referrer' initiates the process; this is the dentist or doctor who prescribes or requests a radiograph. Their responsibility is to provide sufficient clinical information for the next person in the chain - the 'IRMER practitioner' - to be able to justify the radiograph. Training should help these clinicians select appropriate cases for CBCT examination, understanding the risks they seek to expose the patient to, the likely yield from the examination. These dentists should also know how to manipulate and interpret the resulting imaging dataset to extract the required diagnostic information.

The 'IRMER practitioner' is the key person in the chain and they take responsibility in law for the radiographic exposure. This is normally a radiologist within a hospital setting but it may be a dentist in a dental practice. Their role is to justify the exposure, weighing the risks against the benefits and considering the necessity of CBCT and its imaging alternatives. Here training in CBCT capabilities and risks is essential to allow a balanced decision.

The 'operator' is anyone playing any practical role in the radiographic exposure or reporting on the images and must be 'adequately trained' for the role they undertake. The central person here is the person taking the radiograph - this may be a radiographer, dentist, dental nurse or other DCP with a Certificate in Dental Radiography. Training should enable them to carry out the required CBCT examination safely, accurately and with maximum dose optimisation for the intended diagnostic role (see above). The 'ALARA' principle applies here as much as in conventional dental radiography.

The referring dentist also must have a responsibility to ensure that they are making the best possible use of the resulting data and that they are correctly trained and properly supported to do so.

Training is therefore crucial for the whole dental team. It should comprise of training from the manufacturer's CBCT applications specialist on how to use the particular machine, an update on radiation hazards and imaging pitfalls relevant to $\mathrm{CBCT}$, selection criteria for $\mathrm{CBCT}$ imaging and how to approach interpretation of cross-sectional and three-dimensional images.

\section{CONCLUSION}

CBCT technology is increasingly accessible in dental practice. It hugely expands diagnostic and treatment possibilities for patients. However, CBCT should only be used after careful consideration, where conventional two-dimensional imaging techniques are not sufficient or where access to the technological processes such as guided surgery will improve patient management.

When selecting the best CBCT examination for an individual, it is important to minimise X-ray dose while striving for an image that enables appropriate diagnosis and management. This requires an understanding of the concepts behind СВCT and related technologies, making appropriate training essential for every member of the dental team.
The authors would like to thank Sanchia Purkayastha, Cavendish Imaging, London.

1. Whaites E. Periapical radiography. In: Essentials of dental radiology and radiography, $4^{\text {th }}$ ed. Chapter 10. Churchill Livingston Elsevier, 2007.

2. Cohenca N, Simon J H, Roges R, Morag Y, Malfaz J $M$. Clinical indications for digital imaging in dentoalveolar trauma. Part 1: traumatic injuries. Dent Traumato/ 2007; 23: 95-104.

3. Patel S, Dawood A, Whaites E, Pitt Ford T. New dimensions in endodontic imaging: part 1. Conventional and alternative radiographic systems. Int Endod J 2009; 42: 447-462.

4. Gröndahl H-G, Huumonen S. Radiographic manifestations of periapical inflammatory lesions. Endod Top 2004; 8: 55-67.

5. Revesz G, Kundel H L, Graber M A. The influence of structured noise on the detection of radiologic abnormalities. Invest Radiol 1974; 6: 479-486.

6. Kundel H L, Revesz G. Lesion conspicuity, structured noise, and film reader error. AJR Am J Roentgenol 1976; 126: 1233-1238.

7. White S, Pharaoh M. Advanced imaging modalities. Oral radiology: principles and interpretation, 5th ed. Chapter 13. St Louis, Mo: Mosby, 2004.

8. Hounsfield G N. Computerised transverse axial scanning (tomography): Part 1. Description of system. Br J Radiol 1973; 46: 1016-1022.

9. Arai $Y$, Tammisalo E, Iwai K, Hashimoto K, Shinoda K. Development of a compact computed tomographic apparatus for dental use. Dentomaxillofac Radiol 1999; 28: 245-248.

10. Mozzo P, Procacci C, Tacconi A, Martini P T, Andreis I A. A new volumetric CT machine for dental imaging based on the cone-beam technique: preliminary results. Eur Radio/ 1999; 8: 1558-1564.

11. Hashimoto $K_{1}$ Yoshinori $Y$, Iwai $K_{1}$ Araki M, Kawashima $S$ et al. A comparison of a new limited cone beam computed tomography machine for dental use with a multidetector row helical CT machine. Oral Surg Oral Med Oral Pathol Oral Radiol Endod 2003; 95: 371-377.

12. Hashimoto K, Kawashima S, Araki M, Sawada K, Akiyama Y. Comparison of image performance between cone-beam computed tomography for dental use and four-row multidetector helical CT. J Oral Sci 2006; 48: 27-34.

13. Bryant A, Drage N, Richmond S. Study of the scan uniformity from an i-CAT cone beam computed tomography dental imaging system. Dentomaxillofac Radiol 2008; 37: 365-374.

14. Ludlow J B, Davies-Ludlow L E, Brooks S L, Howerton W B. Dosimetry of 3 CBCT devices for oral and maxillofacial radiology: CB Mercuray, NewTom $3 \mathrm{G}$ and i-CAT. Dentomaxillofac Radiol 2006; 35: 219-226.

15. Paloma J M, Hans M G. Influence of CBCT exposure conditions on radiation dose. Oral Surg Oral Med Oral Pathol Oral Radiol Endod 2008; 105: 773-782.

16. IRMER: Statutory Instrument No. 1059. The lonising Radiation (Medical Exposure) Regulations 2000.

17. Verdun F R, Bochud F, Gudinchet F, Aroua A, Schnyder P, Meuli R. Quality initiatives radiation risk; what you should know to tell your patient. Radiographics 2008; 28: 1807-1816.

18. Loubele M, Bogaerts R, Van Dijck E, Pauwels R et al. Comparison between effective radiation dose of CBCT and MSCT scanners for dentomaxillofacial applications. Eur J Radiol 2008; Jul 16 (Epub).

19. Ludlow J B, Davies-Ludlow LE, White S C. Patient risk related to common dental radiographic examinations: the impact of 2007 International Commission on Radiological Protection recommendations regarding dose calculation. J Am Dent Assoc 2008; 139: 1237-1243. 